\title{
On the Field Level Loss of a VHT PPDU in a MIMO-OFDM System for a WiFi Direct 802.11ac WLAN
}

\author{
Gul Zameen Khan*, Ruben Gonzalez ${ }^{\dagger}$, Xin-Wen Wu", Eun-Chan Park ${ }^{\|}$

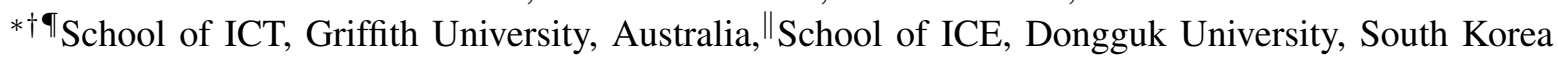 \\ ${ }^{*}$ gz.khan@griffithuni.edu.au, ${ }^{\dagger}$ r.gonzalez@griffith.edu.au, ${ }^{\top}$ X.wu@ griffith.edu.au, ${ }_{\text {ecpark@dongguk.edu }}$
}

\begin{abstract}
This paper proposes a methodology to reduce Packet Error Rate (PER) of a Very High Throughput (VHT) physical layer frame in a WiFi Direct (WD) 802.11ac Wireless Local Area Network (WLAN) by examining the loss of individual fields. To this end, a 2x2 Multiple Input Multiple Output - Orthogonal Frequency Division Multiplexing (MIMO-OFDM) transceiver is implemented under a TGn multipath fading channel D. The system generates VHT Physical Layer Conformance Procedure (PCLP)- Protocol Data Unit (PPDU) frames which are passed through the TGn channel $D$. Thereafter performance of the system is determined by calculating the overall PER. Then the impact of each field of the VHT PPDU is analysed separately in order to estimate their percentage loss in the overall PER. The field level scrutinization of the PER provides a design level insight into the VHT PPDU performance. Based on simulation results, an efficient methodology is proposed to reduce the overall PER without changing the hardware of the current 802.11ac system. In addition, since a detailed packet loss analysis can determine the efficiency of a rate selection algorithm. Therefore the findings of the paper can also be taken into account while designing an efficient rate selection algorithms for the WD 802.11ac WLAN.
\end{abstract}

Keywords-Very High Throughput, PPDU, MIMO, OFDM, WiFi Direct, WLAN, 802.11ac, packet loss

\section{INTRODUCTION}

The current state of the art amendment by IEEE 802.11 standard families is 802.11ac that achieves a Very High Throughput (VHT) up to 6 Giga bit per second (Gbp/sec). The VHT is achieved by employing advanced techniques at Medium Access Control (MAC) and Physical (PHY) layers. At the MAC layer schemes such as larger frame size, dynamic channel allocation, and frame aggregations are used. Similarly, new features are added at the PHY layer that include a wider channel bandwidth with the help of channels bonding, higher order Modulation and Coding Schemes (MCSs), higher number of Multiple Input Multiple Output (MIMO) technologies and a Multi User (MU)-MIMO etc.[1].

A considerable amount of literature has been published on exploring new features of the 802.11ac. In [2], an overview of the key mandatory and optional features of the MAC and PHY layers of $802.11 \mathrm{ac}$ is explored. Thereafter, the MAC throughput of the 802.11ac and 802.11n are compared in terms of frame aggregation techniques. However, the paper does not consider the losses that are resulted from the frame aggregation techniques. Similarly, a comprehensive overview of the underlying principles, implementation details and key enhancing features of the 802.11n and 802.11ac are described in [3]. In particular, the use of MU-MIMO in 802.11ac standard has several potential applications in current and future networks. The authors in [4] present a joint multicast-unicast design using MU-MIMO that sends unicast and multicast streams together by leveraging the unused MIMO capability and link margin thereby achieving improved throughput gain. Similarly, a capacity analysis of the $802.11 \mathrm{ac}$ and $802.11 \mathrm{n}$ is presented in [5] using the Ekahau Site Survey tool. The author recommends the use of wider channels and $5 \mathrm{GHz}$ band to increase the capacity of a user. In our previous work [6], we presented a comprehensive performance analysis of system throughput of the 802.11ac in terms of contention window at MAC layer and MCSs, spatial streams, and channel bandwidth at PHY layer. Subsequently, the system throughput was assessed using a detailed analytical and simulation implementations.

It is an established fact that a packet loss in 802.11 happens either due to a collision or a weak signal strength [7]. In order to differentiate between the two losses, 802.11 uses Distributed Coordination Function (DCF) and the number of retransmissions [8]. In DCF mechanism, the transmission of a successful data frame is identified by the reception of an ACK frame. If the ACK is not received for a particular packet in a specified amount of time, the data packet is considered lost due to collision. On the other hand if the transmission of a data packet is failed after a certain number of retransmissions, then the packet loss is considered due to weak signal. The packet loss in 802.11 has been analysed by a number of researchers. Rayanchu et al. [7] diagnose the packet loss in 802.11 and perform an experiment to identify the causes of the packet loss. They propose an algorithm called COLLIE (Collision Inferencing Engine) to examine the error patterns within the symbols of a PHY layer to differentiate between the losses due to collision and weak signal. Similarly, an analytical approach is adopted in [9] to determine the performance loss due to multipath noise as a function of Orthogonal Frequency Division Multiplexing (OFDM) and channel parameters for a narrowband OFDM system.

WiFi Direct (WD) is a new technology that enables two or more WiFi devices to communicate with each other in the absence of an Access Point (AP) [10]. The devices first forms a group and then communicate. In every group, one device acts as a Group Owner (GO) and the rest of the members join the group as client(s). The GO is responsible for AP like 
activities in the group. WD has many potential applications in future wireless networks and Internet of Things (IoT) [11].

The overall packet loss in 802.11 standards has been substantially analysed in the literature in the context of $802.11 \mathrm{a} / \mathrm{b} / \mathrm{g} / \mathrm{n}$. However, the field level investigation of a packet header in the WD 802.11ac network is yet to be explored for potential improvements. This is important due to two main reasons. First the new standard i.e., 802.11ac amended the VHT PPDU and new fields were added to previous PPDU to achieve VHT. Second, the field level scrutinization is mandatory to get an insight into PER in the WD 802.11ac network. Thus we are motivated to estimate the field level loss of the VHT PDDU in WD 802.11ac network thereby reducing the overall PER. This paper makes the following contributions:

i. It estimates the loss percentage of individual fields in the VHT PPDU in the 802.11ac network.

ii. The PER is investigated in the context of a $2 \times 2$ MIMO-OFDM transceiver which is implemented in Matlab under a TGn multipath fading channel D. Thus the analysis results apply to a specific but practical environment.

iii. A methodology is proposed to reduce the overall PER without changing the hardware of the current WD 802.11ac system.

The remainder of the paper is organized as follows: In Section II, the background knowledge of the VHT PPDU is described. Then the system model is presented in terms of system architecture and simulation set up in Section III. Next, the results are discussed in Section IV. Lastly, conclusions are drawn in Section V.

\section{BACKGROUND}

In this section we describe the basics of a PHY frame in a MIMO-OFDM WD 802.11ac system. When a packet is received by a PHY layer from a MAC layer, it is called a PHY-Layer Protocol Service Data Unit (PSDU). The PHY layer consists of two sub layers namely: Physical Layer Conformance Procedure (PLCP) and Physical Medium Dependent Sublayer (PMD). A VHT preamble i.e., PLCP header and trailer is added to the PSDU which is then called a PCLP Protocol Data Unit (PPDU). Thus the PPDU is a complete PLCP frame, including PLCP headers, MAC headers, MAC data field, and MAC/PLCP trailers. The PPDU is called a VHT PPDU because it achieves high data rate. The field structure for the VHT PPDUs consist of a preamble and data portions. The VHT PPDU also contains legacy preamble fields (i.e., L-STF, L-LTF, and L-SIG) which are common with HT and non-HT format preambles. However, VHT format preamble fields include additional format-specific training and signalling fields. Each format defines a data field for the transmission of a user payload data. The VHT PPDU is shown in TABLE I. We describe each of the fields as follows [1], [12]:

1) L-STF: The Legacy Short Training Field (L-STF) is a common field among VHT, HT, and Non-HT preambles. It consists of 10 short preambles of duration $0.8 \mu$ s each. This preamble is constructed by 12 OFDM subcarriers out of 52 subcarriers to detect the start of a 802.11 packet. The 12 individual subcarriers are modulated with Binary Phase Shift Keying (BPSK) to ensure a low peak to average power ratio. The L-STF can be decoded by any receiver who is compatible with the OFDM technology. It is also used for synchronizing timers and selecting an antenna. Eq. 1 indicates how to calculate the duration of the L-STF, i.e., $T_{S T F}[1]$.

$$
T_{S T F}=10\left(\frac{T_{F F T}}{4}\right)
$$

where $T_{F F T}$ indicates the length of a Fast Fourier Transform (FFT) and is given by Eq. 2 .

$$
T_{F F T}=\frac{1}{\triangle F}
$$

where $\triangle F$ indicates the subcarrier frequency spacing in $\mathrm{kHz}$. For a $20 \mathrm{MHz}$ channel bandwidth under OFDM, the $\triangle F=$ $312.5 \mathrm{kHz}$. Thus $T_{F F T}=3.12 \mu \mathrm{s}$ and $T_{S H O R T}=8 \mu \mathrm{s}$. It can also be calculated for a $40 \mathrm{MHz}$ channel bandwidth and other channel bandwidths [1].

2) L-LTF: The Legacy Training Field (L-LTF) is also part of the VHT, HT, and Non-HT preambles. It is used to estimate the underlying channel, frequency offset and time synchronization. The duration of the L-LTF, i.e., $T_{L T F}$ is computed in Eq. 3 [1].

$$
T_{L T F}=2 . T_{F F T}+T_{G I 2}
$$

where $T_{G I 2}$ indicates a double guard interval which is $1.6 \mu \mathrm{s}$. By using $T_{G I 2}$ and $T_{F F T}$ in Eq. 3 , the $T_{L T F}=8 \mu \mathrm{s}$.

3) L-SIG: The Legacy Signal Field (L-SIG) is used to calculate data rate, length, and parity information. It consists of 24 bits. The L-SIG is a component of the VHT, HT, and non-HT PPDUs. It is transmitted using BPSK modulation with a rate of 1/2 Binary Convolutional Coding (BCC) [12].

4) VHT-SIG-A: The VHT Signal A (VHT-SIG-A) field consists of two symbols: VHT-SIG-A1 and VHT-SIG-A2. The VHT-SIG-A carries information required to interpret VHT PPDUs. Only an 802.11ac system can decode the VHT-SIG-A fields. These fields are modulated and coded using either of 0 to 9 MCS. For a detailed structure of the VHT-SIG-A, [1] can be refereed.

5) VHT-STF: The VHT STF serves the same purpose as the L-STF. Just as the first training fields help a receiver tune in the signal, the VHT-STF assists the receiver in detecting a repeating pattern and setting receiver gain [12].

6) VHT-LTF: The VHT-LTF is used to estimate a MIMO channel and track pilot subcarriers. The VHT-LTF includes one VHT long training symbol for each spatial stream indicated by the selected MCS. Each symbol is $4 \mu \mathrm{s}$ long. The number of OFDM symbols in the VHT-LTF $\left(N_{V H T L T F}\right)$ is derived from the total number of space-time streams i.e., $N_{S T S, \text { total }}$ which is computed in Eq. 4 [1]:

$$
N_{S T S, \text { total }}=\sum_{u=0}^{N_{\text {user }}-1} N_{S T S}(u)
$$

where $N_{\text {user }}$ indicates the total number of users and $N_{S T S}(u)$ is the number of space-time streams per user $u$. It may consist of $1,2,4,6$, or 8 symbols; the number of required symbols 
TABLE I

VHT PPDU FRAME STRUCTURE

\begin{tabular}{|c|c|c|c|c|c|c|c|c|c|c|c|c|}
\hline L-STF & L-LTF & L-SIG & VHT-SIG-A1 & VHT-SIG-A2 & VHT-STF & VHT-LTF1 & $\ldots$ & VHT-LTFN & VHT-SIG-B & SF & VHT-Data & Tail \\
\hline
\end{tabular}

is rounded up to the next highest value, so a link with five streams would require six symbols [12].

7) VHT-SIG-B: The Very High Throughput Signal B (VHTSIG-B) carries information about the data rate, the length of A-MPDU per user and the MIMO for multi-user case. It is transmitted with MCSO and consists of a single OFDM symbol located between the VHT-LTF and the data portion of the VHT format PPDU. The size of the VHT-SIG-B depends on the channel bandwidth and the number of users (SU, MU) [13].

8) Service Field $(S F)$ : The SF is part of the VHT-Data portion. It consists of 16 bits out of which 7 bits are used for scrambler initialization to avoid long runs of identical bits; 1 bit is reserved for future use while 8 bits are used for Cyclic Redundancy Check (CRC) to detect errors in VHT-SIG-B [1].

9) VHT-Data: The VHT-Data field contains a variable size PSDU from the higher layers. The PSDU may contain one or more MAC Protocol Data Unit (MPDU) or several MPDUs in an aggregate MPDU (A-MPDU). The size of the PSDU may vary from 1 to 1048575 bytes [1]. If no Data field is present in the physical layer payload, it is called a Null Data Packet (NDP), which is used by the VHT PHY for setting up beamforming, measurement, and tuning [13].

10) Tail: It is a 6-bit field which is used to terminate a convolutional code. Tail bits are not needed when a LowDensity Parity-Check (LDPC) is used [13].

\section{SySTEM MODEL}

\section{A. System Architecture}

Our system architecture is based on the PHY layer specifications of $802.11 \mathrm{ac}$. The system consists of an OFDM Transmitter (Tx) with 2 transmit chains, a MIMO TGn channel D [14] that represents an office, and an OFDM Receiver (Rx) with two transmit chains. The high level blocks of the Tx, Rx and the channel as well as the subblocks of the Tx and Rx are depicted in Fig. 1. Different fields of the VHT PPDU are sent in the order as shown in TABLE. I. The VHT PPDU is passed through various sub-blocks of the Tx as illustrated in 1. Since the underlying technology that we consider is WD, thus the Tx acts as a GO and the Rx works as a client. The sub blocks of the Tx and Rx are briefly explained in the following subsections.

1) PHY Padding: The first part of the $\mathrm{Tx}$ is Padding in which extra zeroes are added to the data to match the size recommended by [1].

2) Scrambler: The next part is the scrambler which is used to manipulate the PSDU to produce a random signal that makes it difficult to extract the original signal. The detailed properties and structure of the scrambler can be found in [1].

3) BCC Encoder Parser: The BCC encoder parser demultiplexes the scrambled bits into different BCC encoders in a round robin fashion. The number of outputs depends on the number of the Forward Error Correction (FEC) encoders.

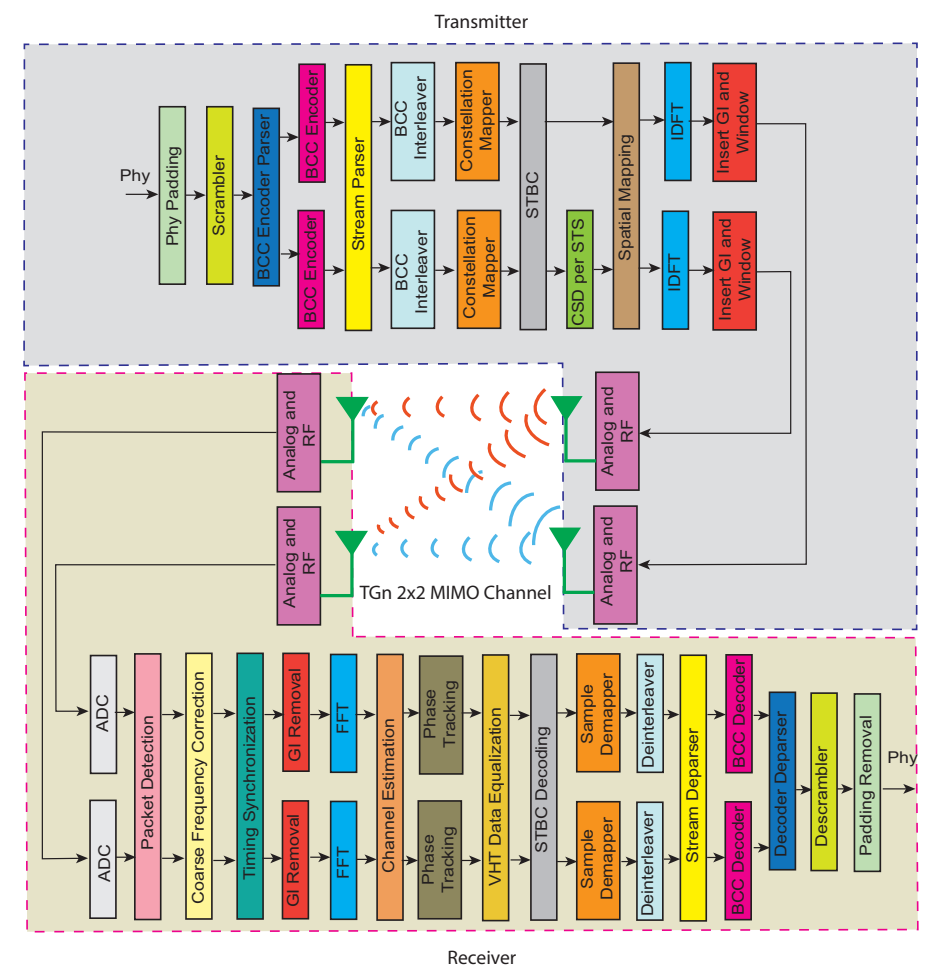

Fig. 1. Throughput for different channels as a function of number of STAs

4) BCC Encoder: The DATA field in the VHT PPDU is encoded using one of the FEC encoding techniques available in the 802.11ac namely: BCC or LDPC. We have used BCC to encode the DATA field.

5) Stream Parser: After coding and puncturing, the data bit streams at the output of the FEC encoders are processed in groups of $N_{C B P S}$ bits where $N_{C B P S}$ indicates the number of the coded bits per OFDM symbol. Each of these groups is rearranged into $N_{S S}$ (number of spatial streams) blocks, which is the first representation of a MIMO streams. This operation is referred to as the stream parsing [1].

6) BCC Interleaver: The bits at the output of the stream parser are processed in groups of $N_{C B P S}$ bits. Each of these groups is divided into $N_{S S}$ blocks of $N_{C B P S}$ bits, and each block shall be interleaved by an interleaver. The interleaving is performed separately for every OFDM symbol. It is carried out in three steps of permutations. The first step ensures that the adjacent codded bits are mapped into non-adjacent subcarriers. The second permutation ensures that the adjacent bits are distributed into less and more significant bits of the constellation. Finally, the third permutation is called frequency rotation which is only applied when more than one spatial streams exist. The equations for permutation are described in [1]. 
7) Constellation Mapper: The bits from the BCC Interleaver are fed into the constellation mapper that maps them to complex constellation points for BPSK, Quadrature Phase Shift Keying (QPSK),16-Quadrature Amplitude Modulation (QAM), 64-QAM and 256-QAM [1].

8) Spatial Time Block Code (STBC): This optional step is used to transmit one spatial stream across multiple antennas for extra redundancy. The space-time block coder takes a single constellation symbol output and maps it onto multiple radio chains, transforming the spatial streams into space-time streams [12].

9) CSD per STC: CSD per STC stands for Cyclic Shift Delay per Space Time Code. A CSD is applied at the Tx to avoid interference among the same streams transmitted along each transmit chain. It is applied for each STC that comes from the STBC block [15].

10) Spatial Mapping: The Spatial Mapper maps the space time streams into transmit chains. We have used a direct mapping technique i.e., a single space time stream is mapped into a single antenna. However, the number of spatial time streams $\left(N_{S T S}\right)$ may not be equal to the number of transmit chains $\left(N_{T x}\right)$ which is a common technique in beam forming [1].

11) IDFT: An Inverse Discrete Fourier Transform is applied to convert the OFDM from frequency domain into the time domain.

12) Insert GI and Window: The guard interval is inserted at the start of each symbol, and each symbol is windowed to improve signal quality at the $\mathrm{Rx}$ [12].

13) $A D C$ : The signal received at each antenna is first sampled and then converted into a digital signal by an Analogue to Digital Converter (ADC).

14) Packet Detection: The start of the VHT PPDU packet is detected with the help of OFTM training symbols in the L-STF. Afterwords, a delay and correlate algorithm is used [16].

15) Coarse Frequency Detection: This block returns a coarse estimate of the Carrier Frequency Offset (CFO) for the VHT PPDU passing through a TGn 2x2 MIMO channel $\mathrm{D}$ given the received time-domain L-STF samples and channel bandwidth [3].

16) Timing Synchronization: The training symbols are used for Tx and Rx timing synchronization [16].

17) Channel Estimation: A VHT transmission has a preamble that contains VHT-LTF symbols, where the data tones of each VHT-LTF symbol are multiplied by the entries belonging to a matrix called $P_{V H T L T F}$, to enable the channel estimation at the Rx [1].

18) Phase Tracking: The multiplication of the pilot tones in the VHT-LTF symbol by the $R_{V H T L T F}$ matrix instead of the $P_{V H T L T F}$ matrix allows Rx to track phase and frequency offset during a MIMO channel estimation using the VHT-LTF [1].

19) VHT Data Equalization: The data field in the VHT PPDU is recovered using Zero Forcing (ZF) equalizer which is a linear equalization algorithm that applies an inverse of the frequency response of the channel to the received signal [18].
TABLE II

CHARACTERISTICS OF MULTIPATH FADING TGN CHANNEL D

\begin{tabular}{|l|l|l|l|l|l|}
\hline Parameter & Value & Parameter & Value & Parameter & Value \\
\hline $\begin{array}{l}\text { Rician } \\
\text { K-factor for } \\
\text { LOS (dB) }\end{array}$ & 3 & $\sigma_{R M S}$ & 50 & $\sigma_{\text {Max }}$ & 390 \\
\hline $\begin{array}{l}\text { Rician } \\
\text { K-factor for } \\
\text { NLOS (dB) }\end{array}$ & $-\infty$ & $\begin{array}{l}\text { Shadow } \\
\text { fading (dB) } \\
\text { before LOS }\end{array}$ & 3 & No of tap & 18 \\
\hline No of clusters & 3 & $\begin{array}{l}\text { Shadow fad- } \\
\text { ing (dB) } \\
\text { after NLOS }\end{array}$ & 4 & $d_{B P}(\mathrm{~m})$ & 10 \\
\hline
\end{tabular}

\section{B. Simulation Setup}

A simulation model based on the system architecture discussed in Section III is developed in Matlab. A VHT PPDU is constructed with all its fields illustrated in TABLE I which is processed by the Tx. Fig. 1 shows the blocks through which the DATA field is passed. Some of the blocks are skipped for the sake of simplicity. The detailed diagrams for all the fields can be found in [1]. A 2x2 MIMO system is used with two independent streams (i.e., $N_{S S}=2$ ) at each antenna. The VHT PPDU is passed through a $2 \times 2$ MIMO multipath fading TGn channel D [14]. The channel specifications are illustrated in TABLE II where $\sigma_{R M S}, \sigma_{M a x}$ and $d_{B P}$ indicate the Root Mean Square (RMS) delay spread, maximum delay, and breakpoint distance, respectively. The channel bandwidth is set to $20 \mathrm{MHz} / 40 \mathrm{MHz}$ and the sampling frequency is set to $20 \mathrm{MHz} / 40 \mathrm{MHz}$. The distance between the $\mathrm{Tx}$ and $\mathrm{Rx}$ is fixed at $10 \mathrm{~m}$ and the distance between the Tx as well as the Rx antennas is $0.5 \lambda$. The length of the PSDU remains 3000 bytes throughout the simulation and extra bits are padded accordingly. After the VHT PPDU passes through the multipath fading $2 \times 2$ MIMO TGn channel D, an Additive White Gaussian Noise (AWGN) is added to the signal. As we consider a WD 802.11ac, thus the carrier frequency is set to $5.25 \mathrm{GHz}$ which is used throughout the simulation. We send a maximum of 1000 packets for each Signal to Noise (SNR) point until a $10 \%$ PER is achieved which is the requirement specified by $802.11 \mathrm{ac}$ standard [1]. We then calculate the average PER for each SNR point.

\section{RESUlTS AND Discussions}

In this section, the results are presented and discussed in detail. First of all, the statistics of the PER are calculated for different MCSs (i.e., MCS0 to MCS8) as a function of SNR in order to investigate the overall PER. Note that the MCS0 in this case indicates BPSK with coding rate of $1 / 2$, $800 \mathrm{~ns}$ GI, and a date rate of $14 \mathrm{Mb} / \mathrm{s}$ under a $2 \times 2 \mathrm{MIMO}$ channel with $20 \mathrm{MHz}$ bandwidth. For more details, Section 22.5 of the standard 802.11ac [1] can be referred. As shown in Fig. 2, the PER can be divided into three levels namely: initial level, intermediate level, and final level. We observe that the PER is $100 \%$ at initial level for all MCSs. However, the range of SNR points for which the PER remains 1 is different for different MCSs . Similarly, the PER reaches a minimum value between 0 and 0.1 at final level for all MCSs. However, the PER follows a decreasing trend from maximum to minimum 


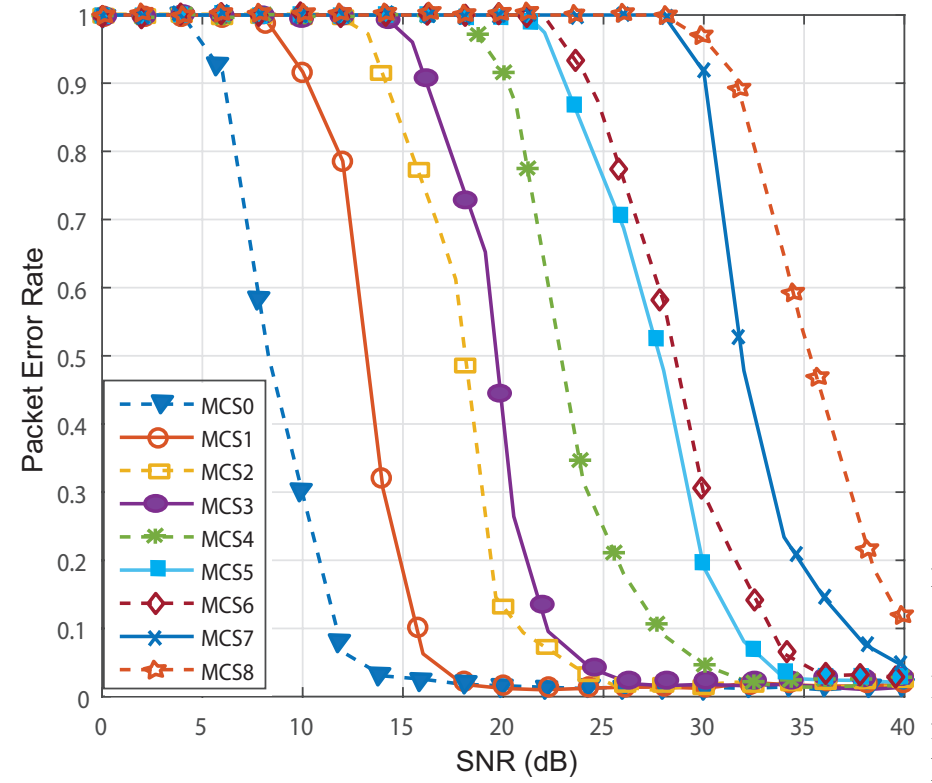

Fig. 2. The PER for different MCSs as a function of SNR values

for intermediate level for all MCSs. The negative slope of a decreasing line is different for each MCS. The range of the SNR points for which the PER falls from a maximum value to minimum (i.e., intermediate level) is smaller than the ranges of SNR points of initial and final levels. As shown in Fig. 2, in case of MCS0, $100 \%$ of the packets are lost when the SNR is less than $4 \mathrm{~dB}$, while the PER reaches $92 \%$ at SNR of $6 \mathrm{~dB}$ and drops to almost $9 \%$ when the SNR reaches $9 \mathrm{~dB}$. The PER becomes 0 after the SNR reaches $15 \mathrm{~dB}$ and stays 0 afterwords in the final level. A similar trend can be observed for other MCSs. The PER is increased as we increase the modulation and coding order i.e., MCSs.

The first field in the VHT-PPDU is L-LTF that helps in detecting the packet. If this field is failed to be correctly decoded then the whole packet has to be sent again even if the rest of the packet reaches the $\mathrm{Rx}$ antennas without any interference. This is because the Tx does not know if there is any transmission arriving at its antennas if the detection fails. The L-LTF helps in detecting the start of a packet. In order to examine how many packets gets failed due to L-LTF, we run the simulations and calculates the PER which occurs due to the failure of the L-LTF. The PER for different MCSs is shown in Fig. 3. It can be seen that the PER ranges from 5\% to $10 \%$ for different MCS at lower SNR $(<25 \mathrm{~dB})$. The average PER does not change much even if we increase the MCS. Thus the MCS0 is the best possible option as it can be decoded by all the previous standards of 802.11 that do not support a VHT PPDU. The PER drops below 5\% at higher SNR ( $>25 \mathrm{~dB})$. It is also interesting to note that the MCS has no significant change on the loss of the L-LTF.

Similarly, PER due to the L-STF for different MCSs is illustrated in TABLE 3. We observe that a major percentage of the PER is due to the loss of the L-LTF at lower SNR. For example, $72.73 \%$ packets are lost due to L-LTF at $2 \mathrm{~dB}$ SNR for MCSO. The PER is 0 when the SNR reaches $6 \mathrm{~dB}$ for

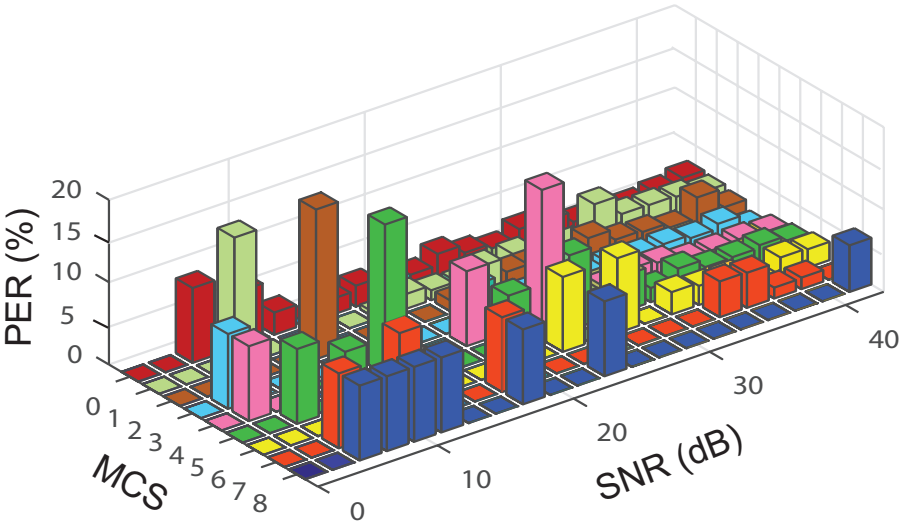

Fig. 3. The PER due to L-LTF field in VHT PPDU

lower MCSs, however there still exists some loss (9\% PER) for higher MCSs at the same SNR value. The PER due to LLTF becomes 0 after the SNR value is increased to $7 \mathrm{~dB}$ and so on. Thus the L-LTF should be taken great care of at SNR less than $6 \mathrm{~dB}$.

TABLE III

PACKET ERROR RATE DUE TO L-STF FIELD IN VHT PPDU

\begin{tabular}{|c|c|c|c|c|c|c|}
\hline \multicolumn{2}{|c|}{ SNR (dB) } & 0 & 2 & 4 & 6 & $\mathrm{SNR}>6 \mathrm{~dB}$ \\
\hline \multirow{2}{*}{ ?̊ } & MCS0 & 90.91 & 72.73 & 27.27 & 0.00 & 0.00 \\
\hline & MCS1 & 100.00 & 63.64 & 54.55 & 0.00 & 0.00 \\
\hline \multirow{2}{*}{ 䒿 } & MCS2 & 90.91 & 81.82 & 9.09 & 0.00 & 0.00 \\
\hline & MCS3 & 100.00 & 63.64 & 45.45 & 18.18 & 0.00 \\
\hline \multirow{2}{*}{ 它 } & MCS4 & 100.00 & 90.91 & 36.36 & 0.00 & 0.00 \\
\hline & MCS5 & 100.00 & 54.55 & 63.64 & 0.00 & 0.00 \\
\hline \multirow{3}{*}{$\frac{\bar{v}}{\frac{v}{0}}$} & MCS6 & 100.00 & 90.91 & 18.18 & 9.09 & 0.00 \\
\hline & MCS7 & 72.73 & 63.64 & 18.18 & 9.09 & 0.00 \\
\hline & MCS8 & 100.00 & 81.82 & 27.27 & 9.09 & 0.00 \\
\hline
\end{tabular}

In Fig. 4-5, we consider the Bit Error Rate (BER) analysis of yet two more important fields of the VHT-PPDU i.e., VHTSIG-A and L-SIG for two different channel bandwidths i.e., $20 \mathrm{MHz}$ and $40 \mathrm{MHz}$ for a $2 \times 2 \mathrm{MIMO}-\mathrm{OFDM}$ system under a TGn channel D model. In Fig. 4, the BER of the VHTSIG-A is around 0.06 and 0.002 at $1 \mathrm{~dB}$ SNR for $20 \mathrm{MHz}$ and $40 \mathrm{MHz}$, respectively. The BER decreases for both the channel bandwidths as the SNR value is decreased until the BER reaches almost 0 for both the bandwidths. However, it can be seen that the BER for the $40 \mathrm{MHz}$ bandwidth channel is almost $97 \%$ better than that of the $20 \mathrm{MHz}$ bandwidth channel.

In the same way, the BER of L-SIG is shown in Fig. 5 as a function of SNR for a $20 \mathrm{MHz}$ and $40 \mathrm{MHz}$ bandwidth channels. The BER is 0.0513 and 0.0012 at SNR $1 \mathrm{~dB}$ for a $20 \mathrm{MHz}$ and a $40 \mathrm{MHz}$ bandwidth channels, respectively. The BER falls as the SNR is increased for both the channel bandwidths. However, the the BER is around 97\% less for $40 \mathrm{MHz}$ bandwidth channel as compared to $20 \mathrm{MHz}$ bandwidth channel. Thus the performance of the system can be improved if $40 \mathrm{MHz}$ channel is used with a $2 \times 2$ MIMO-OFDM system under TGn channel D model.

The simulation results are repeated for all the fields of the VHT PPDU including VHT-LTF and VHT-SIG-B. However, all the results are not included in this paper for the sake of 


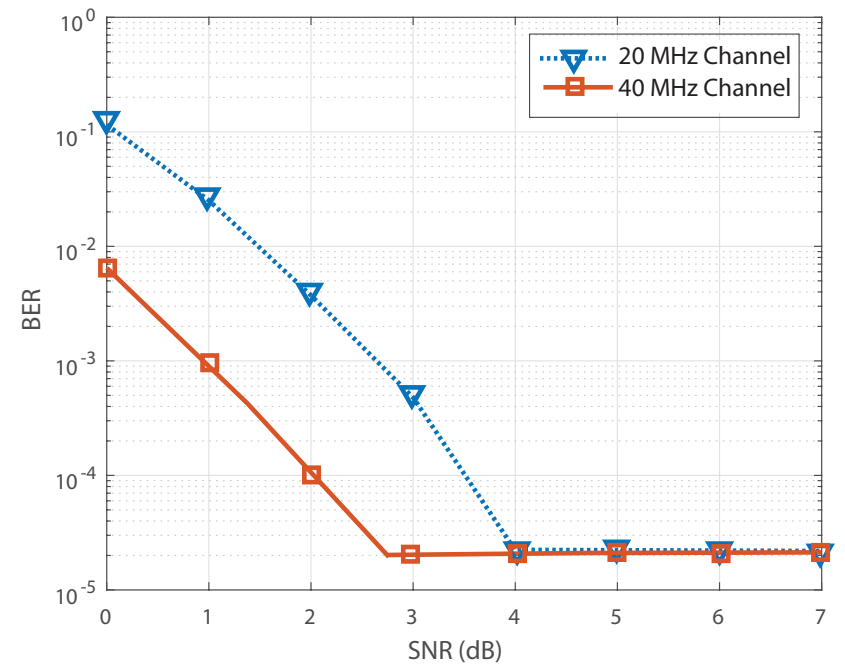

Fig. 4. BER of VHT-SIG-A field in VHT PPDU

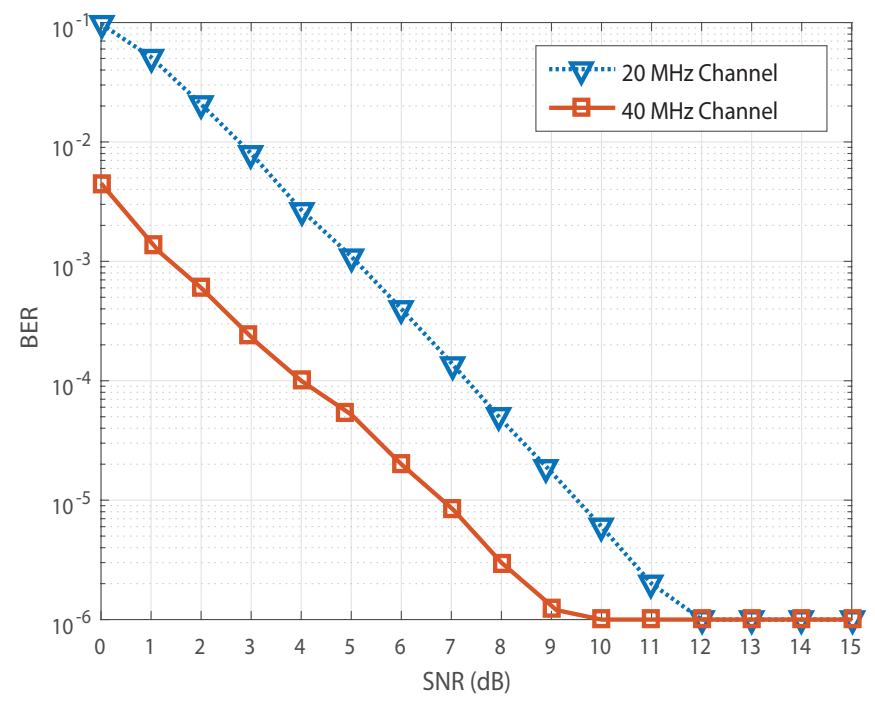

Fig. 5. BER of LSIG field in VHT PPDU

simplicity and due to limitation of the space. The important trends that we have observed throughout the experimentations are the same for all the fields. In a nutshell, the change of MCS does not improve the successful reception of the packet header. However, the use of wider channels for sending the packet header can tremendously decrease the PER and thereby improve the overall performance of the system.

\section{CONClusion}

This paper investigated the field level loss of a VHT PPDU packet in a $2 \times 2$ MIMO-OFDM transceiver under a TGn D model for a WD 802.11ac network. For this purpose, a complete system model is developed and implemented whereby the PER for a complete VHT PPDU as well as the percent PER for individual fields are analysed. Similarly, the BER analysis of different individual fields is examined for different channel bandwidths. It was shown that the system performance can be improved if the individual fields of the VHT PPDU are taken care of separately. The simulation results show that the use of different modulation and coding schemes can affect the overall PER. However, they could not substantially decrease the loss of the individual fields. The BER for individual fields can be decreased tremendously if a wider channel is used for transmissions.

\section{REFERENCES}

[1] Wireless LAN MAC and PHY Specifications - Amendment 4: Enhancements for Very High Throughput for Operation in Bands below $6 \mathrm{GHz}$, IEEE Standard 802.11ac, 2013.

[2] E. H. Ong, J. Kneckt, O. Alanen, Z. Chang, T. Huovinen and T. Nihtil, "IEEE 802.11ac: Enhancements for very high throughput WLANs," in 22nd IEEE International Symposium on Personal, Indoor and Mobile Radio Communications, Toronto, 2011, pp. 849-853.

[3] E. Perahia and R. Stacey, Next Generation Wireless LANs: 802.11n and 802.11ac, 2nd Edition, United Kingdom, Cambridge University Press, 2013.

[4] B. X. Wu, K. C. Lin, K. C. Hsu and H. Y. Wei, "HybridCast: Joint multicast-unicast design for multiuser MIMO networks," in IEEE Conference on Computer Communications (INFOCOM), Kowloon, 2015, pp. 1724-1732.

[5] T. Vanhatupa, "Wi-Fi Capacity Analysis for 802.11 ac and $802.11 \mathrm{n}$ : Theory and Practice," Ekahau Inc., 2013.

[6] G. Z. Khan, R. Gonzalez, E. C. Park, X. W. Wu, "Analysis of Very High Throughput (VHT) at MAC and PHY Layers under MIMO Channel in IEEE 802.11ac WLAN," ICACT Transactions on Advanced Communications Technology, vol. 5, no. 4, pp. 877-888, July, 2016.

[7] S. Rayanchu, A. Mishra, D. Agrawal, S. Saha and S. Banerjee, "Diagnosing Wireless Packet Losses in 802.11: Separating Collision from Weak Signal," The 27th Conference on Computer Communications (INFOCOM), Phoenix, AZ, 2008, pp. 1409-1417.

[8] IEEE Standard for Wireless LAN MAC and PHY Specifications, IEEE Standard 802.11, November, 1997.

[9] F. Heereman, W. Joseph, E. Tanghe, L. Verloock and L. Martens, "Performance Degradation Due to Multipath Noise for Narrowband OFDM Systems: Channel-Based Analysis and Experimental Determination," IEEE Transactions on Wireless Communications, vol. 14, no. 3, pp. 1396-1405, March, 2015.

[10] Wi-Fi Peer-to-Peer (P2P) Technical Specification, ver. 1.0. Wi-Fi Alliance, P2P Technical Group, 2009.

[11] G. Z. Khan, R. Gonzalez, E. C. Park, X. W. Wu, "A reliable multicast MAC protocol for WD 802.11 networks," in European Conference on Networks and Communications (EuCNC), 2015, pp.224-228.

[12] M. Gast, 802.11ac: A Survival Guide, Sebastopol, CA, O'Reilly Media, Inc, 2013.

[13] www.mathwork.com

[14] V. Erceg, L. Schumacher, P. Kyritsi, et al., "TGn Channel Models," ver. 4. IEEE 802.11-03/940r4, May, 2004.

[15] Aruba Networks, 802.11ac In-Depth.[Online]. Available:http://www.arubanetworks.com/pdf/technology/whitepapers/ WP 80211acInDepth.pdf

[16] T. M. Schmidl and D. C. Cox, "Robust frequency and timing synchronization for OFDM," IEEE Transactions on Communications, vol. 45, pp. 16131621, December, 1997.

[17] H. Minn, M. Zeng, V. K. Bhargava, "On timing offset estimation for OFDM systems," IEEE Communications Letters, vol. 4, no. 7, pp. 242244, July, 2000.

[18] J. Mark and W. Zhuang, Wireless Communications and Networking, Prentice Hall, 2003. 